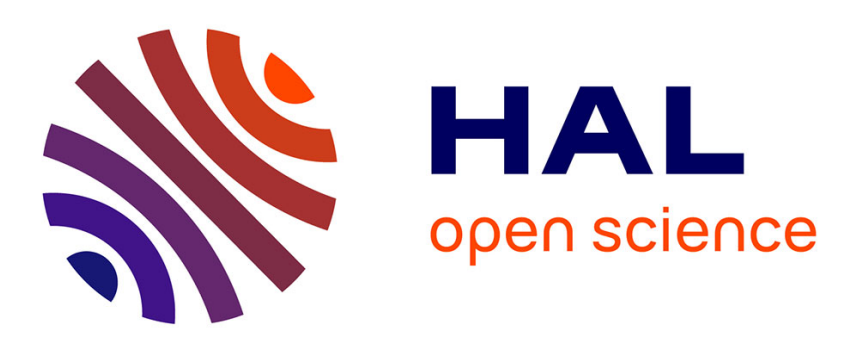

\title{
Ce que le vote par approbation révèle des préférences des électeurs français
}

Isabelle Lebon, Antoinette Baujard, Frédéric Gavrel, Herrade Igersheim, Jean-François Laslier

\section{- To cite this version:}

Isabelle Lebon, Antoinette Baujard, Frédéric Gavrel, Herrade Igersheim, Jean-François Laslier. Ce que le vote par approbation révèle des préférences des électeurs français. Revue Economique, 2017, 68 (6), pp.1063-1076. 10.3917/reco.pr2.0084 . halshs-01420369v2

\section{HAL Id: halshs-01420369 \\ https://shs.hal.science/halshs-01420369v2}

Submitted on 19 Apr 2023

HAL is a multi-disciplinary open access archive for the deposit and dissemination of scientific research documents, whether they are published or not. The documents may come from teaching and research institutions in France or abroad, or from public or private research centers.
L'archive ouverte pluridisciplinaire HAL, est destinée au dépôt et à la diffusion de documents scientifiques de niveau recherche, publiés ou non, émanant des établissements d'enseignement et de recherche français ou étrangers, des laboratoires publics ou privés. 


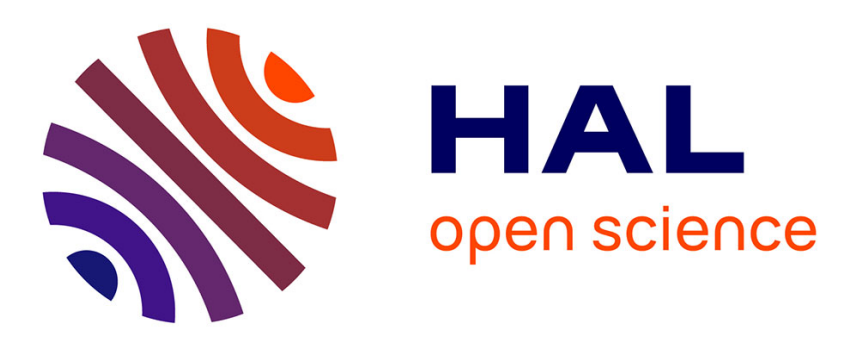

\section{Ce que le vote par approbation révèle des préférences des électeurs français}

Isabelle Lebon, Antoinette Baujard, Frédéric Gavrel, Herrade Igersheim, Jean-François Laslier

\section{- To cite this version:}

Isabelle Lebon, Antoinette Baujard, Frédéric Gavrel, Herrade Igersheim, Jean-François Laslier. Ce que le vote par approbation révèle des préférences des électeurs français . 2016. halshs-01409106

\section{HAL Id: halshs-01409106 \\ https://shs.hal.science/halshs-01409106}

Preprint submitted on 5 Dec 2016

HAL is a multi-disciplinary open access archive for the deposit and dissemination of scientific research documents, whether they are published or not. The documents may come from teaching and research institutions in France or abroad, or from public or private research centers.
L'archive ouverte pluridisciplinaire HAL, est destinée au dépôt et à la diffusion de documents scientifiques de niveau recherche, publiés ou non, émanant des établissements d'enseignement et de recherche français ou étrangers, des laboratoires publics ou privés. 
UMR 5824

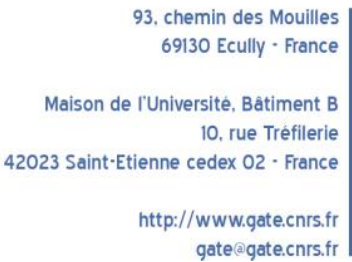

WP 1636- December 2016

\title{
Ce que le vote par approbation révèle des préférences des électeurs français
}

\author{
Isabelle Lebon, Antoinette Baujard, Frédéric Gavrel, Herrade Igersheim, \\ Jean-François Laslier
}

\begin{abstract}
:
Cet article vise à déterminer une répartition, le long d'un axe politique, des candidats à l'élection présidentielle de 2012 qui soit fondée sur les opinions exprimées par les électeurs à travers une expérimentation de vote par approbation, plutôt que sur un positionnement a priori. L'axe endogène représentatif du paysage politique obtenu s'avère correspondre à un axe gauche-droite classique. Cependant, des analyses plus précises du comportement des électeurs remettent en cause l'hypothèse d'unimodalité des préférences politiques qui est généralement associée à cette structure. En particulier, l'attitude qui consiste à approuver simultanément des candidats situés des deux côtés de l'échiquier politique mais sans soutenir les candidats intermédiaires sur l'axe est largement répandue, et nettement plus fréquente chez les hommes que chez les femmes.
\end{abstract}

\section{Keywords:}

Vote, approbation, préférences électorales, unimodalité

JEL codes:

C93, D72

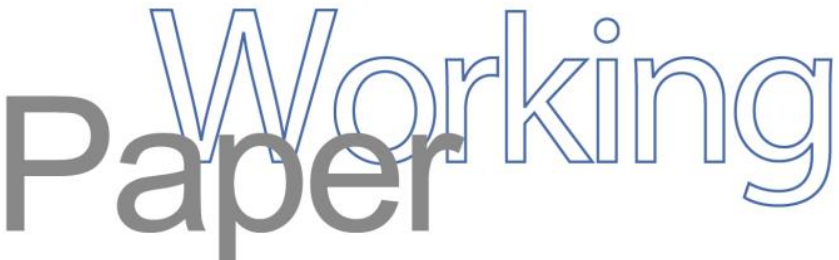




\title{
CE QUE LE VOTE PAR APPROBATION REVELE \\ DES PREFERENCES DES ELECTEURS FRANÇAIS*
}

19 octobre 2016

\author{
Isabelle Lebon ${ }^{1}$, Université de Caen Normandie, CNRS,_CREM UMR 6211 \\ Antoinette Baujard ${ }^{2}$, Univ. Lyon, UJM Saint-Etienne, CNRS, GATE L-SE UMR 5824, \\ Frédéric Gavrel $^{3}$, Université de Caen Normandie, CNRS,_CREM UMR 6211, \\ Herrade Igersheim ${ }^{4}$, CNRS et Beta UMR7522, Université de Strasbourg \\ Jean-François Laslier ${ }^{5}$, CNRS, PJSE UMR $8545^{6}$
}

\begin{abstract}
Résumé
Cet article vise à déterminer une répartition, le long d'un axe politique, des candidats à l'élection présidentielle de 2012 qui soit fondée sur les opinions exprimées par les électeurs à travers une expérimentation de vote par approbation, plutôt que sur un positionnement $a$ priori. L'axe endogène représentatif du paysage politique obtenu s'avère correspondre à un axe gauche-droite classique. Cependant, des analyses plus précises du comportement des électeurs remettent en cause l'hypothèse d'unimodalité des préférences politiques qui est généralement associée à cette structure. En particulier, l'attitude qui consiste à approuver simultanément des candidats situés des deux côtés de l'échiquier politique mais sans soutenir les candidats intermédiaires sur l'axe est largement répandue, et nettement plus fréquente chez les hommes que chez les femmes.
\end{abstract}

\section{What approval voting reveals about the preferences of French voters}

\begin{abstract}
The purpose of this article is to determine a distribution along a political axis of the candidates for the 2012 French presidential election on the basis of the opinions of voters, rather than on a priori ideological positions. To do so, experimental data from a voting experiment on approval voting are used. The obtained endogenous axis representative of the political landscape corresponds to a fairly standard left-right axis. However, a more precise analysis of the behavior of voters questions the unimodality assumption of political preferences usually associated with this structure. Voters often simultaneously approve candidates on both sides of the political spectrum but without supporting intermediary candidates on the axis; besides, this attitude is more common among men than women.
\end{abstract}

Codes JEL : C93, D72

\footnotetext{
* Version préliminaire de l'article à paraître dans la Revue Economique

1 Esplanade de Paix, 14000 Caen ; isabelle.lebon@unicaen.fr ; auteur correspondant.

210 rue Tréfilerie, 42023 Saint-Etienne Cedex 2 ; antoinette.baujard@univ-st-etienne.fr

${ }^{3}$ Esplanade de Paix, 14000 Caen ; frederic.gavrel@unicaen.fr

${ }^{4} 61$ avenue de la Forêt Noire, 67085 Strasbourg Cedex ; igersheim@unistra.fr

${ }^{5} 48$ boulevard Jourdan, 75014 Paris ; jean-francois.laslier@ens.fr

${ }^{6}$ Les auteurs remercient Alpha Oumar Diop pour l'aide qu'il leur a apportée dans le traitement des données et les rapporteurs anonymes pour la pertinence de leurs remarques.
} 


\section{INTRODUCTION}

Les systèmes de vote uninominaux utilisés en France dans la plupart des scrutins officiels, tels que les élections présidentielles ou législatives, contraignent les électeurs dans leur expression puisque, parmi tous les candidats auxquels ils peuvent souhaiter apporter leur voix, ils sont obligés de n'en retenir qu'un. Les résultats des élections ne donnent ainsi qu'une vision très partielle des préférences politiques, qui ne peuvent être appréhendées qu'au travers d'études d'opinion complémentaires, à condition que lesdites études cherchent à aller au-delà de la simple prévision du résultat de la prochaine élection. Au titre de ces études plus approfondies, on peut citer celle réalisée par Michelat et Tiberj [2007] à l'occasion des présidentielles de 2007. Ces auteurs étudient la façon dont les électeurs se positionnent et positionnent les différents candidats dans le paysage politique en invitant les personnes interrogées à se référer à un schéma gauche-droite. Or, la situation de chaque parti politique dans le clivage gauche-droite est une idée suffisamment ancienne et ancrée dans les esprits ${ }^{7}$ pour prédéterminer largement la perception que les électeurs ont des candidats et l'ensemble des réponses qu'ils sont susceptibles de faire dans le cadre d'une telle enquête. Que cette représentation unidimensionnelle gauche-droite soit héritée de l'histoire ne suffit pourtant pas à justifier sa pertinence aujourd'hui. Aussi souhaitons-nous ne pas considérer l'axe gauche-droite comme une hypothèse de travail, mais comme un éventuel résultat qu'il est nécessaire de faire émerger.

Le but de notre contribution est donc de proposer une représentation de l'espace politique français qui n'aurait pas pour origine une vision préconçue des choses, mais les préférences réellement exprimées des électeurs au travers du vote. ${ }^{8}$ Cela suppose évidemment de nous référer à un mode de scrutin qui permette aux électeurs de s'exprimer sur chacun des candidats. Cette condition nous renvoie de façon générale aux procédures de vote par évaluation et, plus particulièrement, au vote par approbation qui invite chaque électeur à répartir l'ensemble des candidats en deux groupes, ceux qu'il soutient et voudrait voir élus d'une part, et ceux qu'il rejette d'autre part, sans que soit limité le nombre des approbations qu'il peut donner.

Le vote par approbation a été longuement étudié dans la littérature théorique, et son application testée à travers différentes sortes d'expérimentations (Brams et Fishburn [1978], Laslier et Sanver [2010], Baujard et Igersheim [2010], Igersheim et al. [2016]). Si les principaux questionnements des expérimentations en laboratoire sont relatifs à la comparaison des résultats agrégés et à l'importance du vote stratégique relativement à d'autres modes de scrutin, les études in situ s'intéressent en outre à l'acceptation, par les votants, de modes de scrutin alternatifs, et à une meilleure représentation de l'offre politique française telle que perçue par les électeurs eux-mêmes. Dans la lignée de ce dernier courant de littérature et nous appuyant sur des données expérimentales recueillies lors du premier tour des présidentielles de 2012 (Baujard et al. [2013]), nous établissons un panorama de l'échiquier politique français tel qu'il ressort des approbations distribuées aux différents candidats par un échantillon d'électeurs. Notre objectif est comparable à celui poursuivi par Laslier [2004], [2006] et Baujard et al. [2011] en ce qu'il revient à construire « une sorte de photographie officielle des préférences des électeurs (...) purement endogène et sans référence à un ensemble de ques-

\footnotetext{
${ }^{7}$ Crapez [1998] fait remonter la naissance de ce concept à la fin du XIXème siècle et sa fixation définitive à l'époque du Front Populaire.

${ }^{8}$ Une autre approche consiste à faire évaluer, par exemple par un panel d'experts, les positions des partis sur différentes questions a priori jugées pertinentes et discriminantes. Cette méthode consiste alors à situer les partis dans un espace défini par l'ensemble des questions retenues. Typiquement, deux dimensions sont nécessaires pour une telle représentation. Voir Laver et al. [2006] pour une étude sur la France.
} 
tions spécifiées a priori $»$ (Laslier [2006], p. 160 et p. 163, traduction). Nous introduisons ici une procédure d'agrégation originale : il s'agit de sélectionner, parmi tous les classements possibles des différents candidats, celui qui est le plus compatible avec les soutiens simultanés que leur ont accordés les électeurs, en ce sens que ledit classement minimise le nombre de candidats non approuvés intercalés entre les candidats approuvés sur l'ensemble des bulletins expérimentaux de notre échantillon.

La méthodologie mise en œuvre et les données utilisées sont présentées dans les deux premières sections. Un axe endogène est déterminé et commenté dans une troisième section. A partir de ce résultat, une quatrième section permet de rechercher et d'expliciter les éventuelles disparités dans le comportement de vote de différents sous-groupes d'électeurs à partir des seules caractéristiques connues que sont le genre et l'âge. Nous identifions ainsi des écarts importants entre les comportements des hommes et des femmes, ainsi qu'entre ceux des 30 ans et moins et des plus de 30 ans.

\section{DU VOTE PAR APPROBATION A LA DEFINITION D’UN AXE POLI- TIQUE. METHODOLOGIE}

Une procédure de vote par approbation offre aux électeurs la possibilité d'apporter leurs suffrages à plusieurs des candidats présents. Plus précisément, un participant au scrutin peut soutenir simultanément tous les candidats qu'il souhaiterait voir élus, au lieu de devoir n'en choisir qu'un seul, comme dans les procédures uninominales. Il s'agit donc d'un mode de scrutin à un seul tour dont le gagnant est le candidat qui recueille le plus grand nombre d'approbations. Cependant, notre objectif n'est pas ici d'identifier le vainqueur désigné par ce mode de scrutin, mais d'observer les associations entre candidats que font implicitement les électeurs à travers les soutiens qu'ils expriment.

En votant par approbation, les électeurs partagent de fait l'ensemble des candidats proposés entre ceux avec lesquels ils se sentent en accord et voudraient voir élus et ceux qu'ils rejettent. A travers cette distinction, chaque électeur est amené à exprimer son opinion sur l'offre politique existante, c'est-à-dire qu'en fonction de ses propres préférences politiques, chaque électeur détermine les candidats qu'il juge suffisamment proches de lui pour les soutenir. Indirectement, chacun exprime sa propre vision du paysage politique, une vision dans laquelle les candidats approuvés simultanément se trouvent logiquement proches les uns des autres.

Encore faut-il pouvoir légitimement penser que les électeurs expriment leurs véritables préférences à travers le vote par approbation. C'est l'idée soutenue par Brams et Fishburn [1983] qui expliquent que les électeurs peuvent décider de soutenir un nombre plus ou moins important de candidats mais qu'ils étendent toujours la liste des approuvés dans l'ordre de leur préférence. Pour Niemi [1984], cette latitude donnée aux électeurs de déterminer librement le nombre de leurs soutiens fait du vote par approbation une incitation à un comportement stratégique. Cependant, la stratégie en question ne conduit pas les électeurs à travestir leurs opinions et ne remet donc pas réellement en cause la fiabilité des préférences individuelles révélées par cette procédure.

En revanche, il est possible d'imaginer des cas où un électeur rationnel peut avoir strictement intérêt à soumettre un bulletin d'approbation contredisant ses véritables préférences (voir De Sinopoli et al. [2006]). Mais de telles situations semblent très peu plausibles, notamment quand le nombre d'électeurs est grand (Laslier [2009]). Or l'élection présidentielle au suffrage universel est évidemment l'exemple type d'un électorat très vaste. D'où notre confiance dans le fait que les électeurs expriment globalement leurs véritables préférences lorsque la règle d'approbation leur est proposée. Il serait pourtant précipité d'en conclure 
directement qu'aucun électeur n'a jamais écarté le moindre candidat appartenant à l'un des partis politiques dont il se sent proche. Mais il n'y a pas de raison d'y voir une décision « stratégique »; il est plus probable qu'un tel choix relève d'un effet de valence, c'est-à-dire du rejet individuel de certains candidats, un effet inhérent à la personnalisation du scrutin lors de l'élection présidentielle. Cette restriction ne constitue pas un élément suffisant pour remettre globalement en cause l'usage sincère de la règle d'approbation et donc l'intérêt de l'exercice.

L'agrégation des préférences exprimées par les électeurs à travers le vote par approbation permet donc de redéfinir une structure de l'espace politique, non plus à partir d'une conception a priori du positionnement des partis auxquels ces candidats appartiennent ou en référence à des questions de sociétés supposées a priori pertinentes, mais à partir des associations de candidats faites par les électeurs. Le but de cette agrégation est d'établir l'axe susceptible de traduire au mieux ces associations. Différant en cela de Laslier [2006] et de Baujard et al. [2011] qui recourent l'un à une analyse en composantes principales, les autres à une analyse en composantes multiples, nous proposons ici une méthode originale basée sur la minimisation du nombre de candidats non approuvés intercalés entre les candidats approuvés sur l'ensemble des bulletins de vote constituant notre échantillon. Le classement des candidats en lice issu de cette minimisation sera interprété comme étant l'axe le plus pertinent relativement aux préférences énoncées par les électeurs.

Pour comprendre la signification d'un tel calcul, prenons l'exemple d'un scrutin auquel se présentent 4 candidats $\{A ; B ; C ; D\}$ sur lesquels se sont prononcés par approbation 3 électeurs qui ont émis les bulletins suivants : $\{A ; C ; D\},\{B ; D\}$ et $\{A ; D\}$. L'information contenue dans ces bulletins donne la possibilité d'étudier le bien-fondé des deux axes représentés au graphique 1 au regard de notre hypothèse d'unimodalité au sens large des préférences des agents qui nous conduit à privilégier le classement des candidats qui permet le minimum de candidats non soutenus intercalés entre les candidats approuvés.

L'axe 1, $\{A-B-C-D\}$, ne reflète que très imparfaitement les préférences des électeurs. Dans le premier bulletin, $\{A ; C ; D\}$, le candidat $B$ non approuvé est intercalé entre deux candidats approuvés; dans le deuxième bulletin, $\{B ; D\}, C$ est intercalé entre les deux candidats soutenus; dans le troisième bulletin, $\{A ; D\}, B$ et $C$ sont intercalés entre les deux candidats que l'électeur a choisi. Soit, sur l'ensemble des trois bulletins, un total de quatre candidats non approuvés intercalés entre les candidats approuvés.

Graphique 1 : Deux exemples de classement des candidats A, B, C et D

Axe 1

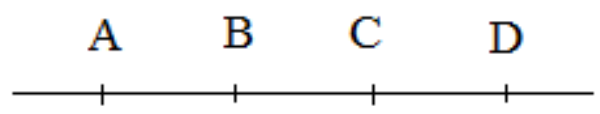

$\underline{\text { Axe } 2}$

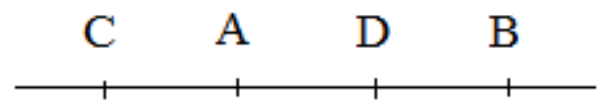

En revanche, le deuxième axe proposé, $\{C-A-D-B\}$, est parfaitement cohérent avec les choix des électeurs, puisque, quel que soit le bulletin considéré, aucun candidat non approuvé ne vient jamais s'interposer entre deux candidats approuvés. Dans notre exemple, cet axe traduit donc de façon satisfaisante la vision des proximités politiques qui est celle des électeurs eux-mêmes, puisque les trois bulletins proposés sont strictement unimodaux sur cet axe. Il en serait évidemment de même de l'axe exactement symétrique $\{B-D-A-C\}$ qui lui est équivalent.

Dans la pratique, l'axe le plus cohérent avec la vision des électeurs ne pourra être obtenu qu'en testant tous les axes issus de l'ensemble des permutations possibles des candidats sur les résultats d'approbation disponibles. Pour $m$ candidats et en tenant compte de 
l'équivalence des classements symétriques, il existe ainsi $\frac{\mathrm{m} !}{2}$ axes possibles dont les scores devront être calculés, le score d'un axe en particulier étant égal au nombre moyen de candidats non approuvés intercalés entre les candidats approuvés par bulletin. L'axe politique retenu comme le plus compatible avec les préférences des électeurs est alors celui qui obtient le score le plus faible. Cette méthode va nous permettre d'obtenir de façon endogène une image du paysage politique français à l'époque des présidentielles de 2012.

\section{PROTOCOLE ET DONNEES EXPERIMENTALES}

Lors du premier tour des élections présidentielles de 2012, une expérimentation de vote a été menée in situ dans cinq bureaux de vote de trois villes françaises : deux bureaux à Strasbourg, deux bureaux à Louvigny (près de Caen), et un bureau à Saint-Etienne (voir Baujard et al. [2013] pour plus de détails sur le protocole qui a été mis en œuvre). Il s'agissait de tester plusieurs procédures de vote alternatives pour comprendre l'impact des modes de scrutin sur le résultat même des élections. Le vote par approbation a ainsi été testé sur l'ensemble des bureaux, et ce sont ces données que nous allons utiliser pour déterminer l'axe politique issu des choix exprimés par les électeurs.

Nous disposons d'un total de 1289 bulletins d'approbation émis par des électeurs ayant accepté de nous indiquer aussi leur vote officiel. La distribution de ces votes officiels n'étant pas conforme aux résultats du premier tour des présidentielles, une pondération des bulletins est mise en place afin de corriger ce biais et d'obtenir un échantillon représentatif (voir la description de la méthode de correction dans Baujard et al. [2014]). Notons cependant que, parmi les bulletins disponibles, seuls ceux comportant l'approbation d'au moins deux candidats ont un impact sur le classement. En effet, les bulletins ne comportant qu'une seule approbation sont unimodaux quel que soit l'axe proposé et, de ce fait, sont non discriminants entre les différents classements possibles. C'est donc sur les bulletins indiquant plusieurs soutiens et sur les préférences politiques qu'ils traduisent que va porter notre article. Il faudra néanmoins garder à l'esprit que $25 \%$ des électeurs de notre échantillon corrigé ont opté pour une seule approbation et ne sont donc pas concernés par les comportements de coapprobation que nous décrivons.

Dans ces conditions, nous disposons de 959 bulletins utilisables pour comprendre comment les électeurs ont associé les candidats du premier tour des élections présidentielles de 2012. La quasi-totalité $(98,77 \%)$ de ces bulletins expriment des choix cohérents entre le scrutin officiel et le vote par approbation, au sens où le candidat sélectionné au vote officiel fait partie des candidats approuvés. Un tel constat incite à penser que la règle d'approbation a été bien comprise par les électeurs.

Les données disponibles permettent d'ordonner sur un axe l'ensemble des candidats en présence, à l'exclusion d'un très petit candidat, Jacques Cheminade, qui n'a obtenu que peu de voix $(0,25 \%)$ au scrutin officiel et a reçu trop peu de soutiens dans nos bulletins d'approbation pour pouvoir espérer obtenir un résultat significatif. Restent donc neuf candidats dont la liste, dans l'ordre du Conseil Constitutionnel, est la suivante : Eva Joly, Marine Le Pen, Nicolas Sarkozy, Jean-Luc Mélenchon, Philippe Poutou, Nathalie Arthaud, François Bayrou, Nicolas Dupont-Aignan et François Hollande. Dans les tableaux et graphiques suivants, ces candidats sont désignés par leurs initiales. Tel est notamment le cas dans le tableau 1 qui donne, pour les 959 bulletins «utiles» de l'échantillon, la proportion des électeurs ayant approuvé chacun des candidats. 
Tableau 1 : Part des électeurs ayant approuvé chacun des candidats (en \%)

\begin{tabular}{|c|c|c|c|c|c|c|c|c|}
\hline EJ & MLP & NS & JLM & PP & NA & FB & NDA & FH \\
\hline 35,29 & 28,41 & 40,4 & 50,12 & 17,5 & 10,52 & 50,13 & 13,7 & 59,7 \\
\hline
\end{tabular}

Chaque candidat apparaît dans 10 à $60 \%$ des bulletins disponibles et généralement son nom est associé à celui de deux autres candidats, puisque, sur les 959 bulletins considérés, le nombre moyen d'approbations est de 3,06. Notons que les choix exprimés par les électeurs qui n'émettent qu'une seule approbation, sont de nature assez différente quant à la répartition des soutiens entre les candidats. Les quatre «petits» candidats, E. Joly, Ph. Poutou, N. Arthaud et N. Dupont-Aignan, ne recueillent en effet qu'environ 1\% chacun de ces approbations uniques. J.-L. Mélenchon et F. Bayrou qui apparaissent dans la moitié des bulletins de multiapprobations, n'obtiennent respectivement que $6 \%$ et $7 \%$ des mono-approbations. Les électeurs qui ont choisi de ne pas multiplier les soutiens ont donc opté en priorité pour F. Hollande $(19 \%)$, M. Le Pen $(23 \%)$ et surtout N. Sarkozy $(41 \%)^{9}$. Si cette distribution des voix peut s'avérer importante sur les résultats électoraux issus d'une règle de vote par approbation, elle n'impacte en revanche pas la construction d'un axe politique.

\section{UN AXE POLITIQUE CLASSIQUE MAIS SANS UNIMODALITE DES PREFERENCES}

Le calcul des scores sur l'ensemble des axes issus de toutes les permutations possibles des 9 candidats retenus fait apparaître l'ordre représenté au graphique 2 comme le plus conforme aux associations faites par les électeurs dans le cadre du vote par approbation. Par convention, nous positionnons à gauche (resp. à droite) de la figure, les candidats usuellement classés à gauche (resp. à droite) de l'échiquier politique.

Graphique 2 : Axe caractéristique des choix exprimés par les électeurs lors des élections présidentielles de 2012

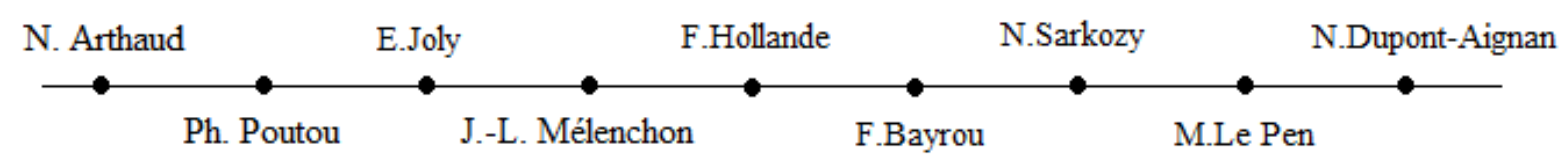

Pour cet axe, le plus cohérent avec les préférences exprimées par les électeurs, le nombre moyen par bulletin de candidats non approuvés intercalés entre les candidats approuvés est de 0,69. En supposant que cette variable suive une loi normale et en estimant l'écart-type à partir de celui qui est observé sur notre échantillon, l'intervalle de confiance autour de cette moyenne s'établit à $[0,59 ; 0,78]$ au seuil de $5 \%$. Un seul autre axe apparaît comme non significativement différent de notre meilleur axe. Cet axe qui obtient un score de 0,73 correspond à l'ordre gauche-droite suivant: N. Arthaud, Ph. Poutou, E. Joly, F. Hollande, J.-L. Mélenchon, F. Bayrou, N. Sarkozy, M. Le Pen et N. Dupont-Aignan. Ce second axe ne présente qu'une seule inversion de positions par rapport à l'axe du graphique 2 entre les candidats F. Hollande et J.-L. Mélenchon.

Nous reviendrons ultérieurement sur les raisons qui conduisent à ne pas exclure une telle inversion, mais, à ce stade, il est important de souligner la très grande robustesse du

\footnotetext{
${ }^{9}$ Voir Baujard et al. [2014] pour une analyse approfondie de la perception des candidats par les électeurs susceptible d'éclairer les différences observées.
} 
classement gauche-droite des candidats, puisque celui-ci reste vérifié dans un cas comme dans l'autre. Les choix des électeurs semblent donc largement intégrer et reproduire une vision a priori de la vie politique française. Notons que pour Laslier [2006] et Baujard et al. [2011], l'axe le plus explicatif retraçait également un classique schéma gauche-droite.

Il ne faut pourtant pas en conclure qu'il n'existe que des électeurs « de gauche » qui n'approuveraient que des candidats placés d'un seul côté de l'axe et que leurs choix viendraient interclasser, et des électeurs « de droite » qui détermineraient les positions relatives de l'autre côté de l'axe. La réalité est nettement plus nuancée. L'élément qui d'emblée incite à douter d'une polarisation politique de l'ensemble des électeurs, est le score assez élevé de notre meilleur axe, puisqu'il reste en moyenne par bulletin 0,69 candidat non approuvé intercalé entre les candidats approuvés. Il en découle logiquement que l'hypothèse d'une unimodalité généralisée des préférences des électeurs ne peut être retenue. Celle-ci supposerait en effet que, pour le classement adéquat des candidats, les électeurs n'approuvent que des candidats contigus sur l'axe. Or, 39,33\% des bulletins ne sont pas unimodaux pour notre meilleur axe. Ces résultats suffisent pour comprendre que, même si l'axe obtenu est très conforme à notre idée préconçue du paysage politique français, les opinions exprimées par les électeurs n'en sont pas simples pour autant.

Pour mettre en perspective les observations précédentes à travers les associations de candidats faites par les électeurs, le tableau 2 représente la matrice croisée des approbations. Il s'agit d'indiquer parmi les soutiens de chacun des candidats en ligne la part de ceux qui ont aussi approuvé les candidats en colonne.

Tableau 2 : Fréquences d'approbations croisées (en \%)

\begin{tabular}{|l|c|c|c|c|c|c|c|c|c|}
\hline & EJ & MLP & NS & JLM & PP & NA & FB & NDA & FH \\
\hline EJ & 100 & 8,05 & 12,0 & 68,1 & 31,65 & 20,19 & 36,65 & 7,61 & 80,3 \\
\hline MLP & 9,99 & 100 & 67,46 & 25,34 & 10,78 & 6,34 & 43,43 & 24,95 & 31,87 \\
\hline NS & 10,48 & 47,44 & 100 & 20,38 & 4,03 & 2,44 & 65,53 & 18,29 & 27,95 \\
\hline JLM & 47,95 & 14,37 & 16,43 & 100 & 26,03 & 17,36 & 37,63 & 9,62 & 80,3 \\
\hline PP & 63,82 & 17,5 & 9,29 & 74,56 & 100 & 39,17 & 31,94 & 12,47 & 73,46 \\
\hline NA & 67,72 & 17,11 & 9,37 & 82,7 & 65,16 & 100 & 31,31 & 17,34 & 77,96 \\
\hline FB & 25,8 & 24,61 & 52,8 & 37,62 & 11,15 & 6,36 & 100 & 16,22 & 51,46 \\
\hline NDA & 19,6 & 51,73 & 53,91 & 35,19 & 15,92 & 13,31 & 59,33 & 100 & 36,07 \\
\hline FH & 47,47 & 15,17 & 18,91 & 67,41 & 21,53 & 13,74 & 43,22 & 8,28 & 100 \\
\hline
\end{tabular}

Lecture : 9,99\% des électeurs qui ont approuvé MLP ont aussi approuvé EJ.

Le premier constat est que, même si les fréquences sont très différentes d'un cas à l'autre, toutes les combinaisons possibles entre candidats sont observées dans les bulletins constituant notre échantillon. Quelques tendances majeures se dégagent de l'analyse du tableau 2. Ceux qui soutiennent les "petits » candidats de gauche - "petits » au sens où ils n'ont obtenu que peu de voix au scrutin officiel -, à savoir N. Arthaud, Ph. Poutou et E. Joly, approuvent dans leur majorité les « gros » candidats situés du même côté du paysage politique. Environ les trois-quarts de leurs électeurs approuvent en effet aussi J.-L. Mélenchon et F. Hollande. Les électeurs du «petit» candidat de droite, N. Dupont-Aignan, en font autant, bien que dans une moindre mesure, vis-à-vis de M. Le Pen, N. Sarkozy et du centriste F. Bayrou $^{10}$. Cependant, dans un cas comme dans l'autre, et bien que ces «petits » candidats

\footnotetext{
${ }^{10}$ Notons que ce constat est conforme à l'une des conclusions formulées par Baujard et al. [2011] dans le con-
} 
soient placés aux extrémités de l'axe politique, une partie non négligeable de leurs électeurs approuvent aussi des candidats de l'autre bord. En prenant l'exemple des soutiens de N. Arthaud et Ph. Poutou, il apparaît qu'environ 9\% d'entre eux accordent aussi leur suffrage à N. Sarkozy, $17 \%$ à M. Le Pen et $31 \%$ à F. Bayrou. N. Dupont-Aignan est lui aussi approuvé par les soutiens de ces « petits » candidats de gauche, N. Arthaud et Ph. Poutou, dans des proportions respectivement égales à $17 \%$ et $12 \%$. Il faut cependant se garder de toute surinterprétation des co-approbations des " petits » candidats de gauche et droite, car chacune des paires concernées n'apparaît au final que dans un peu moins de $2 \%$ des bulletins.

Une dernière remarque, à propos du «petit» candidat de droite N. Dupont-Aignan. On pourrait s'étonner de le retrouver à la droite de M. Le Pen, aussi bien du fait de nos préjugés sur le paysage politique français qu'en constatant que les électeurs ayant approuvé N. Dupont-Aignan soutiennent plus souvent les candidats de gauche que ceux qui ont approuvé $\mathrm{M}$. Le Pen. La position relative de ces deux candidats s'explique par un biais inhérent à la méthode qui peut avoir un effet centrifuge pour les "petits » candidats. En effet, il est moins coûteux, en termes de nombre moyen de candidat non approuvés intercalés, de décentrer un candidat peu approuvé comme N. Dupont-Aignan (13,7\%, cf. tableau 1) qu'une candidate plus souvent approuvée comme M. Le Pen $(28,4 \%)^{11}$.

Les résultats concernant les candidats les plus importants, au sens où ils ont recueilli une part plus substantielle des voix au scrutin officiel, à savoir J.-L. Mélenchon, M. Le Pen, N. Sarkozy, F. Bayrou et F. Hollande, sont également intéressants. Les soutiens croisés entre ces candidats sont fréquents. Même si ces co-approbations existent avant tout entre les candidats du même camp (c'est-à-dire J.-L. Mélenchon et F. Hollande d'un côté, N. Sarkozy et M. Le Pen de l'autre), les votes transversaux sont aussi très élevés. Parmi les résultats les plus marquants, il faut noter d'une part que près d'un soutien sur cinq de F. Hollande opte aussi pour N. Sarkozy et que, dans le sens inverse, c'est le cas de plus d'un électeur sur quatre ; et, d'autre part, ceux qui approuvent M. Le Pen, approuvent aussi F. Hollande pour 31\% d'entre eux. Les fréquences croisées d'approbation mettent en lumière la situation spécifique de $\mathrm{F}$. Bayrou. Ses soutiens approuvent en effet N. Sarkozy et F. Hollande dans des proportions identiques et plus souvent J.-L. Mélenchon que M. Le Pen. Il parait difficile dans ces conditions de l'étiqueter comme appartenant à l'un des deux camps.

Le tableau 2 permet également de comprendre pourquoi une inversion des positions de F. Hollande et de J.-L. Mélenchon sur l'axe ne peut être exclue. Cette éventualité, qui pourrait étonner étant données les opinions politiques défendues par l'un et l'autre, s'explique pourtant aisément. La structure des soutiens croisés entre J.-L. Mélenchon et les candidats de droite est assez proche de celle de F. Hollande bien que les fréquences soient un peu plus faibles. La «distance » de Mélenchon aux candidats de droite n'est donc pas beaucoup plus élevée que celle de F. Hollande. Quand on ajoute à cela le fait que les électeurs des "petits » candidats de gauche ont approuvé F. Hollande dans une proportion notablement plus forte que J.-L. Mélenchon, il ne faut pas s'étonner de ne pas obtenir un résultat plus tranché concernant les positions relatives des deux « gros » candidats de gauche. L'interclassement de F. Hollande et de J.-L. Mélenchon sur l'axe politique de 2012 dépend en effet de l'équilibre

texte des élections présidentielles de 2007 : même si des proximités existent entre les électeurs de droite (alors, N. Sarkozy) et d'extrême-droite (alors, J.-M. Le Pen, Ph. de Villiers), elles sont nettement plus fortes entre les électeurs de gauche (alors, S. Royal) et d'extrême-gauche (alors, O. Besancenot, A. Laguiller).

${ }^{11}$ La position d'E. Joly à la gauche de J.-L. Mélenchon, bien qu'elle soit également susceptible de surprendre, ne relève pas du même type d'effet centrifuge. La situation relative de ces deux candidats s'explique simplement par le fait qu'E. Joly est un peu plus souvent associée aux candidats placés tout à fait à la gauche de l'axe, N. Arthaud et Ph. Poutou, et un peu moins souvent associée aux candidats du centre et de la droite que ne l'est J.-L. Mélenchon. 
entre ces deux forces qui tendent à les rapprocher respectivement des candidats placés à leur droite et à leur gauche.

Finalement, il apparaît que l'axe gauche-droite très classique qui ressort de l'analyse des co-approbations s'accompagne de la part des électeurs d'une vision beaucoup moins dichotomique de la vie politique que ce résultat initial aurait pu le laisser supposer. Un chiffre illustre particulièrement bien ce constat et s'inscrit au cœur des questionnements actuels sur la nécessité ou non d'une recomposition du paysage politique français : parmi ces électeurs qui ont souhaité approuver plusieurs candidats, $25,3 \%$ ont choisi de soutenir simultanément des candidats de gauche et de droite, F. Bayrou étant exclu de l'analyse du fait de son positionnement particulier. Il est donc clair qu'une partie significative de l'électorat ne se situe plus dans une classique vision gauche-droite de la vie politique.

\section{DISPARITES DANS LA PERCEPTION DE L'AXE GAUCHE-DROITE : EFFETS DE GENRE ET D'AGE}

L'axe politique ayant été établi, nous nous interrogeons désormais sur l'existence, à l'intérieur de la population étudiée, de disparités dans la perception de l'axe gauche-droite entre différents sous-groupes d'électeurs. Nous partageons ainsi notre échantillon initial en deux sous-populations composées d'une part des femmes, d'autre part des hommes. Ces deux sous-populations sont de taille similaire et le nombre d'approbations moyen par bulletin ne diffère pas de ce qui est observé dans l'échantillon total.

La première façon de pointer un éventuel écart entre les comportements des deux sousgroupes est de déterminer pour chacun d'eux le score de notre meilleur axe. Les résultats sont éloquents : pour les femmes, le nombre moyen de candidats non approuvés intercalés entre les candidats approuvés n'est que de 0,6 , alors qu'il est de 0,77 pour les hommes. Ces résultats sont significativement différents au seuil de $5 \%$.

Une autre différence intéressante entre les comportements des hommes et des femmes apparaît grâce à une analyse étendue aux autres classements potentiels des 9 candidats. Les deux meilleurs axes sont les mêmes que ceux calculés sur l'ensemble des bulletins, et le deuxième axe ne peut être considéré comme significativement différent du premier ni sur la sous-population des femmes, ni sur celle des hommes. Cependant, le score du deuxième meilleur axe met en évidence une divergence entre les choix des unes et des autres. Dans le cas des femmes, son score est égal à 0,6 comme celui du meilleur axe. Alors que pour les hommes, le nombre moyen de candidats non approuvés du deuxième axe qui s'établit à 0,83 , est donc nettement supérieur à celui du meilleur axe, quand bien même cet écart ne peut pas être considéré comme significatif au seuil de 5\%.

Un premier élément est susceptible de donner une intuition sur l'origine de l'écart observé entre les hommes et les femmes sur le score du meilleur axe, il s'agit de l'importance des soutiens simultanés de candidats de gauche et de droite (hors F. Bayrou). En effet, cette situation concerne $32,65 \%$ des bulletins d'hommes contre seulement $21,47 \%$ des bulletins de femmes. Pour une analyse plus précise des disparités dans le comportement de coapprobation, il faut se référer au tableau 3 qui détaille les soutiens croisés exprimés respectivement par les femmes et les hommes.

Tous les écarts significatifs dessinent une même tendance. Les femmes associent plus fréquemment des candidats situés du même côté de l'échiquier politique. Ainsi les approbations de J.-L. Mélenchon et de F. Hollande sont-elles plus fréquentes chez les soutiens de $\mathrm{Ph}$. Poutou pour les femmes que pour les hommes, de même que les approbations d'E. Joly et de J.-L. Mélenchon sont plus fréquentes pour celles qui ont approuvé F. Hollande. A l'inverse, les hommes optent relativement plus souvent pour des votes transversaux. Parmi les électeurs 
qui ont choisi d'apporter plusieurs approbations, 32\% des hommes qui ont soutenu N. Sarkozy ont aussi approuvé F. Hollande, soit $10 \%$ de plus que chez les femmes. De même que les hommes qui se sont prononcés pour $\mathrm{Ph}$. Poutou sont beaucoup plus nombreux à approuver aussi M. Le Pen. La fréquence importante de soutiens simultanés à des candidats situés à l'opposé les uns des autres sur l'axe politique pour un nombre d'approbations identique explique ainsi le nombre moyen élevé de candidats non approuvés intercalés entre les candidats approuvés que nous observons pour les hommes.

Tableau 3 : Fréquences d'approbation croisées des femmes et des hommes (en \%)

\begin{tabular}{|c|c|c|c|c|c|c|c|c|c|}
\hline & EJ & MLP & NS & JLM & PP & NA & FB & NDA & $\mathrm{FH}$ \\
\hline EJ & 100 & $\begin{array}{l}F: 7,3 \\
H: 9,0\end{array}$ & $\begin{array}{c}\mathrm{F}: 9,0 \\
\mathrm{H}: 15,7\end{array}$ & $\begin{array}{l}F: 68,6 \\
H: 67,2\end{array}$ & $\begin{array}{l}F: 31,9 \\
H: 31,7\end{array}$ & $\begin{array}{l}F: 19,9 \\
H: 20,7\end{array}$ & $\begin{array}{l}F: 34,4 \\
H: 38,8\end{array}$ & $\begin{array}{l}\mathrm{F}: 5,7 \\
\mathrm{H}: 9,6\end{array}$ & $\begin{array}{l}F: 83,5 \\
H: 76,7\end{array}$ \\
\hline MLP & $\begin{array}{l}F: 11,7 \\
\mathrm{H}: 8,8\end{array}$ & 1 & $\begin{array}{l}F: 66,1 \\
H: 68,4\end{array}$ & $\begin{array}{l}F: 27,6 \\
H: 23,7 \\
\end{array}$ & $\begin{array}{l}F: 71,7 \\
H: 13,4\end{array}$ & $\begin{array}{c}F: 58,3 \\
H: 6,7\end{array}$ & $\begin{array}{l}F: 44,6 \\
H: 42,5\end{array}$ & $\begin{array}{l}F: 22,3 \\
H: 26,9 \\
\end{array}$ & $\begin{array}{l}\mathrm{F}: 33,4 \\
\mathrm{H}: 30,7\end{array}$ \\
\hline NS & $\begin{array}{c}\mathrm{F}: 9,7 \\
\mathrm{H}: 11,1\end{array}$ & $\begin{array}{l}F: 44,3 \\
H: 50,0\end{array}$ & 100 & $\begin{array}{l}F: 22,5 \\
H: 18,7\end{array}$ & $\begin{array}{l}F: 4,1 \\
H: 4,0\end{array}$ & $\begin{array}{l}F: 3,6 \\
H: 1,5\end{array}$ & $\begin{array}{l}F: 64,7 \\
H: 66,2\end{array}$ & $\begin{array}{l}\mathrm{F}: 14,0^{*} \\
\mathrm{H}: 21,7^{*}\end{array}$ & $\begin{array}{l}F: 22,5^{*} \\
H: 32,3^{*}\end{array}$ \\
\hline JLM & $\begin{array}{l}\mathrm{F}: 48,9 \\
\mathrm{H}: 46,5\end{array}$ & $\begin{array}{l}F: 10,2 \\
H: 16,8\end{array}$ & $\begin{array}{l}F: 15,0 \\
H: 18,3\end{array}$ & 100 & $\begin{array}{l}F: 26,5 \\
H: 25,6\end{array}$ & $\begin{array}{l}F: 17,2 \\
H: 17,6\end{array}$ & $\begin{array}{l}F: 37,1 \\
H: 37,9\end{array}$ & $\begin{array}{c}\mathrm{F}: 7,8 \\
\mathrm{H}: 11,6\end{array}$ & $\begin{array}{l}\mathrm{F}: 82,7 \\
\mathrm{H}: 77,6\end{array}$ \\
\hline PP & $\begin{array}{l}\mathrm{F}: 72,5^{*} \\
\mathrm{H}: 55,7^{*}\end{array}$ & $\begin{array}{l}\mathrm{F}: 10,2^{*} \\
\mathrm{H}: 24,3^{*}\end{array}$ & $\begin{array}{l}\mathrm{F}: 8,6 \\
\mathrm{H}: 9,9\end{array}$ & $\begin{array}{l}\mathrm{F}: 84,7^{*} \\
\mathrm{H}: 65,1^{*}\end{array}$ & 100 & $\begin{array}{l}F: 36,9 \\
H: 41,3\end{array}$ & $\begin{array}{l}F: 32,9 \\
H: 31,0\end{array}$ & $\begin{array}{l}F: 15,1 \\
H: 10,0\end{array}$ & $\begin{array}{l}\mathrm{F}: 82,4^{*} \\
\mathrm{H}: 65,1^{*}\end{array}$ \\
\hline NA & $\begin{array}{l}\mathrm{F}: 71,8 \\
\mathrm{H}: 63,5\end{array}$ & $\begin{array}{l}\mathrm{F}: 13,2 \\
\mathrm{H}: 21,2\end{array}$ & $\begin{array}{c}F: 12,3 \\
H: 6,4 \\
\end{array}$ & $\begin{array}{l}\mathrm{F}: 87,0 \\
\mathrm{H}: 78,2 \\
\end{array}$ & $\begin{array}{l}F: 58,4 \\
H: 72,2 \\
\end{array}$ & 100 & $\begin{array}{l}F: 34,4 \\
H: 26,1 \\
\end{array}$ & $\begin{array}{l}\mathrm{F}: 13,5 \\
\mathrm{H}: 21,3\end{array}$ & $\begin{array}{l}F: 83,1 \\
H: 72,6\end{array}$ \\
\hline FB & $\begin{array}{l}F: 26,9 \\
H: 24,3 \\
\end{array}$ & $\begin{array}{l}F: 21,9 \\
H: 27,4\end{array}$ & $\begin{array}{l}\mathrm{F}: 47,3^{*} \\
\mathrm{H}: 58,4^{*}\end{array}$ & $\begin{array}{l}F: 40,7 \\
H: 34,3\end{array}$ & $\begin{array}{l}F: 11,3 \\
H: 11,0\end{array}$ & $\begin{array}{l}F: 7,5 \\
H: 5,3\end{array}$ & 100 & $\begin{array}{l}F: 13,3 \\
H: 18,9 \\
\end{array}$ & $\begin{array}{l}F: 50,9 \\
H: 52,1\end{array}$ \\
\hline NDA & $\begin{array}{l}F: 21,2 \\
H: 18,0 \\
\end{array}$ & $\begin{array}{l}F: 52,3 \\
\text { H:51,8 } \\
\end{array}$ & $\begin{array}{l}F: 49,0 \\
H: 57,4\end{array}$ & $\begin{array}{l}F: 40,8 \\
H: 31,3 \\
\end{array}$ & $\begin{array}{l}F: 24,8 \\
H: 10,7\end{array}$ & $\begin{array}{l}F: 14,0 \\
H: 13,0\end{array}$ & $\begin{array}{l}F: 63,4 \\
H: 56,5\end{array}$ & 100 & $\begin{array}{l}F: 35,6 \\
H: 35,8\end{array}$ \\
\hline $\mathrm{FH}$ & $\begin{array}{l}\mathrm{F}: 51,8^{*} \\
\mathrm{H}: 42,7^{*}\end{array}$ & $\begin{array}{l}F: 13,0 \\
H: 17,6\end{array}$ & $\begin{array}{l}F: 13,0^{*} \\
H: 25,3^{*}\end{array}$ & $\begin{array}{l}\mathrm{F}: 72,0^{*} \\
\mathrm{H}: 62,4^{*}\end{array}$ & $\begin{array}{l}F: 22,4 \\
H: 20,6\end{array}$ & $\begin{array}{l}F: 14,3 \\
H: 13,1\end{array}$ & $\begin{array}{l}F: 40,3 \\
H: 46,2\end{array}$ & $\begin{array}{c}\mathrm{F}: 5,9^{*} \\
\mathrm{H}: 10,6^{*}\end{array}$ & 100 \\
\hline
\end{tabular}

Pour chaque type de fréquence, un test de significativité des écarts entre des proportions calculées sur des échantillons indépendants est effectué, les proportions significativement différentes au seuil de $5 \%$ pour les femmes et pour les hommes sont indiquées par un *.

Concernant spécifiquement les femmes, les fréquences présentées au tableau 3 permettent aussi de comprendre pourquoi les scores des deux meilleurs axes sont similaires, un résultat qui signifie que l'inversion entre F. Hollande et J.-L. Mélenchon est sans effet sur le nombre moyen de candidats non approuvés intercalés entre les candidats soutenus. Tout d'abord, leurs approbations croisées atteignent un niveau très important chez les électrices, puisque $72 \%$ des soutiens de F. Hollande approuvent aussi J.-L. Mélenchon. Or, deux candidats qui auraient exactement le même ensemble d'électeurs, pourraient voir leurs positions relatives inversées sur l'axe sans aucun effet sur le score obtenu. Ensuite, nous n'observons que deux différences substantielles dans les proportions dans lesquelles les deux "gros " candidats de gauche sont associés aux autres candidats : (1) celles qui approuvent E. Joly soutiennent nettement plus souvent F. Hollande (83,5\%) que J.-L. Méléchon $(68,6 \%)$, il en résulte un nombre de non-approbations intercalées plus élevé quand $\mathrm{F}$. Hollande est à la droite de J.-L. Mélenchon ; (2) celles qui approuvent F. Bayrou soutiennent plus souvent F. Hollande (50,9\%) que J.-L. Méléchon (40,7\%), il en résulte un nombre de non-approbations intercalées plus élevé quand F. Hollande est à la gauche de J.-L. Mélenchon. Manifestement, 
ces deux effets se compensent, d'où des scores quasi identiques pour les deux meilleurs axes.

Si le partage de l'échantillon entre les sous-populations d'hommes et de femmes est celui qui fait apparaître les différences de comportement les plus claires, un autre résultat mérite d'être également souligné. En constituant deux groupes formés, d'une part, des électeurs de 30 ans et moins et, d'autre part, des plus de 30 ans $^{12}$, il n'existe pas d'écart significatif concernant le score de notre meilleur axe. En revanche, un certain nombre de soutiens croisés peuvent être considérés comme non identiques au seuil de $5 \%$ et quelques tendances nettes peuvent ainsi être établies. Les plus de 30 ans associent souvent l'approbation de N. Sarkozy à l'approbation de N. Dupont-Aignan ou du candidat du centre, F. Bayrou. Au contraire, les jeunes associent plus souvent N. Sarkozy à M. Le Pen (parmi les plus de 30 ans, les soutiens de N. Sarkozy approuvent à 23\% N. Dupont-Aignan, 69\% F. Bayrou et 32\% M. Le Pen, alors qu'ils sont respectivement $10 \%, 52 \%$ et $57 \%$ chez les 30 ans et moins). Une façon d'interpréter ces chiffres pourrait être de dire que pour les 30 ans et moins, le vote M. Le Pen semble devenir un vote politique de droite standard, alors que pour les plus âgés, il continue de relever davantage du vote de protestation ou d'une volonté d'alternance. Ainsi, parmi les plus de 30 ans, 37\% de ceux qui approuvent M. Le Pen soutiennent aussi F. Hollande, alors qu'ils ne sont que 19\% chez les 30 ans et moins. Cela suggère là encore une normalisation du vote $M$. Le Pen en tant que vote de droite chez les plus jeunes.

\section{CONCLUSION}

Au-delà du vainqueur qu'ils pourraient désigner, les résultats d'une procédure de vote par approbation permettent d'appréhender la manière dont les électeurs considèrent l'offre politique qui leur est faite. En effet, un tel mode de scrutin offre aux électeurs la possibilité de voter simultanément pour autant de candidats qu'ils le souhaitent sans affaiblir le soutien qu'ils apportent à chacun d'eux. Dans ces conditions, on peut s'attendre à ce que les participants indiquent leur véritable opinion sur un paysage politique fait de candidats qu'ils souhaiteraient voir élus parce qu'ils représentent correctement leurs idées et de candidats qu'au contraire ils rejettent.

A partir de données expérimentales d'approbation recueillies lors du premier tour de la présidentielle de 2012, nous avons pu reconstituer l'axe politique le plus cohérent avec les choix que les électeurs français ont exprimés à cette occasion. L'axe qui apparaît est conforme à la vision standard d'un classement gauche-droite des candidats à l'élection présidentielle, un classement qui peut être considéré comme très robuste.

Cependant, si la logique politique classique est respectée au niveau agrégé, il serait erroné de conclure que tous les électeurs ont des préférences politiques unimodales. L'existence d'un nombre significatif de "trous » le long de l'axe entre les candidats coapprouvés infirme cette hypothèse d'unimodalité. L'analyse montre aussi que tous les électeurs n'appartiennent pas non plus à un camp bien déterminé en rejetant systématiquement les candidats de l'autre camp, et ce, d'autant plus qu'ils en sont plus éloignés sur l'axe. Ils sont au contraire nombreux à approuver en même temps des candidats habituellement considérés comme de gauche et de droite. Ainsi, les soutiens de tous les candidats placés à droite de F. Hollande tant sur l'axe proposé au graphique 2 que dans les représentations habituelles du paysage politique français sont de un quart à un tiers à voter aussi pour ce dernier. Et ce phénomène de vote transversal ne se limite pas à l'approbation des candidats modérés de l'autre bord, toutes les combinaisons de soutiens croisées sont observées, d'une extrémité à l'autre

\footnotetext{
${ }^{12}$ L'ensemble des deux sous-populations ne correspond pas exactement à notre échantillon initial, certains électeurs ayant choisi de ne pas nous indiquer leur âge.
} 
de l'axe. Ce comportement pourrait s'interpréter comme l'expression d'une contestation visà-vis des partis de gouvernement, lorsqu'il se traduit par la co-approbation des candidats extrêmes des deux bords. Nous mettons en évidence que ce comportement est plus marqué chez les hommes que chez les femmes.

L'existence d'une part importante de bulletins transversaux, sans approbation de candidats intermédiaires, constitue une réelle incitation à opter comme nous l'avons fait pour un critère d'unimodalité au sens large pour déterminer l'axe politique susceptible de traduire au mieux les préférences des électeurs. En effet, un schéma établi sur la base d'une unimodalité stricte qui suppose de retenir l'axe compatible avec l'unimodalité du plus grand nombre de bulletins, occulterait une partie substantielle de l'information dans la mesure où les choix retracés dans les bulletins non unimodaux ne seraient alors absolument plus pris en compte. Clairement, ce sont les bulletins des hommes qui seraient le plus souvent écartés le cas échéant. Il pourrait être intéressant dans des travaux ultérieurs de comparer les axes issus de ces deux visions de l'unimodalité.

Notre étude fait également ressortir un effet de génération. Chez les plus jeunes des électeurs, le vote pour M. Le Pen semble perdre son caractère protestataire pour s'inscrire dans un schéma politique de droite normal. De futures expérimentations devraient nous permettre de vérifier si ces résultats reflètent des tendances durables.

\section{BIBLIOGRAPHIE}

BAUJARD A. et IGERSHEIM H. [2010], « Framed-field experiments on approval voting. Lessons from the 2002 and 2007 French Presidential elections », dans J.-F. LASLIER et R. SANVER (dir.), Handbook on Approval Voting, Heidelberg, Springer, p. 357-395.

BAUJARD A., IGERSHEIM H., GAVREL F., LASLIER J.-F. et LEBON I. [2013], « Vote par approbation, vote par note. Une expérimentation lors de l'élection présidentielle du 22 avril $2012 »$, Revue Economique, 64, p. 177-188.

BAUJARD A., IGERSHEIM H., GAVREL F., LASLIER J.-F. et LEBON I. [2014], «Who's favored by evaluative voting? An experiment conducted during the 2012 French presidential election », Electoral Studies, 34, p.131-145.

BAUJARD A., IGERSHEIM H., et SENNÉ T. [2011], «Political supply in the 2007 French Presidential election: An analysis based on experimental data », Annales d'Economie et de Statistiques, 101-102, p. 142-178.

BRAMS S.J. et FISHBURN P.C. [1978], «Approval voting », American Political Science Review, 72, p. 831-847.

BRAMS S.J. et FISHBURN P.C. [1984], Approval voting, Boston, Birkhauser.

CRAPEZ M. [1998] "De quand date le clivage gauche/droite en France ? ", Revue Française de Science Politique, 48, p. 42-75.

DE SINOPOLI F., DUTTA B. et LASLIER J.-F. [2006], " Approval voting: three examples ». International Journal of Game Theory 35, p. 27-38.

IGERSHEIM H., BAUJARD A. et LASLIER J.-F. [2016], « La question du vote. Expérimentations en laboratoire et In Situ ». Actualité Economique, à paraître, 2016.

LASLIER J.-F. [2004], Le vote et la règle majoritaire. Analyse mathématique de la politique, Paris, CNRS Editions.

LASLIER J.-F. [2006], « Spatial approval voting », Political Analysis, 14, p. 160-185.

LASLIER J.-F. [2009], "The leader rule: A model of strategic approval voting in a large electorate ", Journal of Theoretical Politics, 21(1), p. 113-136. 
LASLIER J.-F. et SANVER R. (dir.) [2010], Handbook on Approval Voting, Heidelberg: Springer, 2010.

LAVER M., BENOIT K. et SAUGER N. [2006], « Policy competition in the 2002 French legislative and presidential elections ». European Journal of Political Research, 45 (4), p. $667-$ 697.

MICHELAT G. et TIBERJ V. [2007], « Gauche, centre, droite et vote : Permanence et mutation d'une opposition », Revue Française de Science Politique, 57, p.371-392.

NIEMI R.G. [1984], "The problem of strategic behavior under approval voting », American Political Science Review, 78, p. 952-958. 